\title{
Introduction to the focus issue: Fifty years of chaos: Applied and theoretical
}

\section{$\operatorname{AUTHOR}(\mathrm{S})$ :}

Hikihara, Takashi; Holmes, Philip; Kambe, Tsutomu; Rega, Giuseppe

\section{CITATION:}

Hikihara, Takashi ... [et al]. Introduction to the focus issue: Fifty years of chaos: Applied and theoretical. Chaos 2012, 22(4): 047501.

\section{ISSUE DATE:}

2012-12-14

URL:

http://hdl.handle.net/2433/187944

\section{RIGHT:}

(C) 2012 American Institute of Physics. This article may be downloaded for personal use only. Any other use requires prior permission of the author and the American Institute of Physics. 


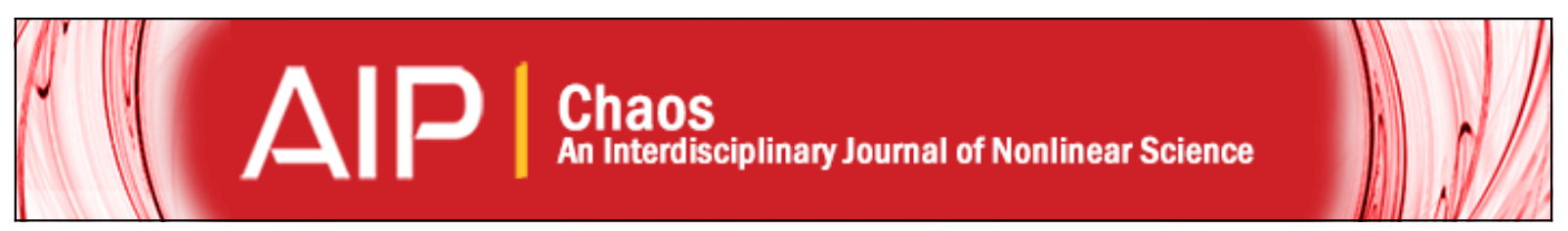

Introduction to the focus issue: Fifty years of chaos: Applied and theoretical

Takashi Hikihara, Philip Holmes, Tsutomu Kambe, and Giuseppe Rega

Citation: Chaos: An Interdisciplinary Journal of Nonlinear Science 22, 047501 (2012); doi: 10.1063/1.4769035

View online: http://dx.doi.org/10.1063/1.4769035

View Table of Contents: http://scitation.aip.org/content/aip/journal/chaos/22/4?ver=pdfcov

Published by the AIP Publishing

\section{Articles you may be interested in}

Introduction to the Focus Issue: Chemo-Hydrodynamic Patterns and Instabilities

Chaos 22, 037101 (2012); 10.1063/1.4756930

Introduction to Focus Issue: Synchronization and Cascading Processes in Complex Networks

Chaos 21, 025101 (2011); 10.1063/1.3605467

Introduction to Focus Issue: Synchronization in Complex Networks

Chaos 18, 037101 (2008); 10.1063/1.2985139

Introduction to Focus Issue: Mixed Mode Oscillations: Experiment, Computation, and Analysis

Chaos 18, 015101 (2008); 10.1063/1.2903177

Introduction: The Fermi-Pasta-Ulam problem-The first fifty years

Chaos 15, 015101 (2005); 10.1063/1.1889345

\section{AlP Re-register for Table of Content Alerts}




\title{
Introduction to the focus issue: Fifty years of chaos: Applied and theoretical
}

\author{
Takashi Hikihara, ${ }^{1}$ Philip Holmes, ${ }^{2}$ Tsutomu Kambe, ${ }^{3, a)}$ and Giuseppe Rega ${ }^{4}$ \\ ${ }^{1}$ Department of Electrical Engineering, Kyoto University, Kyoto 615-8510, Japan \\ ${ }^{2}$ Program in Applied and Computational Mathematics and Department of Mechanical and Aerospace \\ Engineering, Princeton University, Princeton, New Jersey 08544, USA \\ ${ }^{3}$ Department of Physics, University of Tokyo, Tokyo 153-8505, Japan \\ ${ }^{4}$ Dipartimento di Ingegneria Strutturale e Geotecnica, Universita di Roma La Sapienza, 00197 Roma, Italy
}

(Received 8 November 2012; accepted 9 November 2012; published online 14 December 2012)

\begin{abstract}
The discovery of deterministic chaos in the late nineteenth century, its subsequent study, and the development of mathematical and computational methods for its analysis have substantially influenced the sciences. Chaos is, however, only one phenomenon in the larger area of dynamical systems theory. This Focus Issue collects 13 papers, from authors and research groups representing the mathematical, physical, and biological sciences, that were presented at a symposium held at Kyoto University from November 28 to December 2, 2011. The symposium, sponsored by the International Union of Theoretical and Applied Mechanics, was called 50 Years of Chaos: Applied and Theoretical. Following some historical remarks to provide a background for the last 50 years, and for chaos, this Introduction surveys the papers and identifies some common themes that appear in them and in the theory of dynamical systems. (C) 2012 American Institute of Physics.
\end{abstract}

[http://dx.doi.org/10.1063/1.4769035]

Deterministic chaos is, at first glance, surprising. How can the solution of a nonlinear differential equation, or a sequence generated by iterating a smooth map, be so unpredictable? Both are uniquely determined by the initial conditions and, respectively, the equation or the map. We now know that the apparent unpredictability is due to sensitive dependence on initial conditions caused by rapid divergence of neighboring solutions, a property that is quite common in nonlinear differential equations with three or more variables, invertible maps in two or more dimensions, and all non-invertible maps. (The logistic equation is a prime example.) Chaotic dynamics is locally expansive in one or more directions in phase space and contractive in the remaining dimensions. Chaos was first discovered and studied by the mathematician Henri Poincaré in 1889-1912 and it remained a primarily mathematical phenomenon until the 1960s. During this "first period" a substantial theory, built on analysis, geometry and 200 years of work in differential equations and classical mechanics, was developed to characterize invariant sets that live in the phase spaces of nonlinear systems, and to describe bifurcations in which they appear, disappear, or change their stability types. Chaos occupies an important place in this theory of dynamical systems, but it is only a part of it. As described below, some mathematicians drew on examples from the physical sciences and engineering during the first period, but vigorous interactions throughout the physical and mathematical sciences began to develop only in the 1960s and 1970s. The last 50 years has seen a second period flourish, and applications expand to include the biological and life sciences. Chaotic invariant sets can form boundaries for basins of attraction of simple equilibria or periodic orbits, and they can be attracting sets themselves. They can describe irregular

\footnotetext{
${ }^{a)}$ Former Professor, University of Tokyo.
}

time series and spatial structures observed in nature, they can generate quasirandom bit sequences for technological applications, and they appear in analog to digital converters. Analytical, geometrical, and computational methods have been developed to detect and characterize chaotic sets, and experiments have confirmed that they appear in a variety of real systems. The papers in this Focus Issue provide examples of these and other manifestations of chaos.

\section{A BRIEF HISTORY OF CHAOS: 1889-1961 AND A LITTLE BIT BEYOND}

This focus issue grew out of a symposium sponsored by the International Union of Theoretical and Applied Mechanics (IUTAM) that brought together researchers from the physical and biological sciences, engineering, and mathematics to discuss recent developments in nonlinear dynamics and chaos theory. The meeting was timed to celebrate a remarkable discovery, and held 50 years to the week after it was made. In the Department of Electrical Engineering at Kyoto University, on November 27, 1961, a graduate student named Yoshisuke Ueda noticed that orbits of a periodically forced nonlinear oscillator displayed a "randomly transitional" behavior in certain parameter ranges, instead of the periodic, sub- or superharmonic and quasi-periodic motions that he (and his supervisors) expected. ${ }^{1}$ Before surveying the papers that follow, we provide a background by outlining some key work on dynamical systems prior to Ueda's discovery.

Dynamical systems theory began with the work of Poincaré (1854-1912) on the three-body problem of celestial mechanics, ${ }^{2}$ and specifically in a massive paper, ${ }^{3}$ which won a prize celebrating the 60th birthday of King Oscar II of 
Sweden and Norway. In this and his earlier papers, Poincaré proposed new methods for studying nonlinear ordinary differential equations (ODEs). He described the use of first return (Poincaré) maps for the study of periodic motions, defined stable and unstable manifolds, discussed stability issues, developed perturbation methods, and proved the (Poincaré) recurrence theorem. While revising his prize paper, ${ }^{3}$ he realised that certain differential equations describing mechanical systems with two or more degrees of freedom were not integrable in the classical sense, due to the presence of "doubly asymptotic" points, now called homoand heteroclinic orbits. Moreover, he saw that these orbits had profound implications for the stability of motion in general, and realized that his previous claim that a version of the restricted three-body problem of celestial mechanics had only stable behavior was false. In December 1889 and January 1890 , he created the first explicit example of deterministic chaos. ${ }^{4,5}$

G. D. Birkhoff (1884-1944) was one of relatively few mathematicians to continue on Poincaré's path in the early 20th century. ${ }^{6,7}$ Birkhoff's work on iterated mappings of the annulus $^{8,9}$ was especially relevant to the study of periodically forced oscillators to which Ueda's advisor, C. Hayashi, had directed him. Indeed, the van der Pol equation, a model of the vacuum tube diode, played a central role in the development of dynamical systems theory. This began in the 1920s with a brief paper by van der Pol and van den Mark, ${ }^{10}$ engineers at the Phillips Laboratories in Eindhoven, who were interested in subharmonic solutions and who noted in passing that their experimental apparatus produced "an irregular noise" in certain frequency ranges: perhaps an early observation of chaos? Cartwright and Littlewood alluded to this paper in their proof of "discontinuous recurrent" orbits in the van der Pol equation, ${ }^{11}$ and drew on Birkhoff's proof that annulus maps with coexisting stable orbits of distinct periods also possessed complicated invariant sets. Their analysis was later simplified by Levinson. ${ }^{12}$ In the same period, Soviet researchers defined structurally stable systems ${ }^{13}$ (roughly speaking, those that preserve their qualitative properties under small perturbations of the defining ODEs) and began to study bifurcations in planar systems. ${ }^{14,15}$

When Smale became interested in dynamical systems in 1959-1960, ${ }^{16}$ he conjectured that a structurally stable ODE could possess only finite sets of periodic orbits in any bounded region of its state space. Levinson suggested that Cartwright-Littlewood paper might provide a counterexample. Smale's geometric interpretation of a Poincaré map for the forced van der Pol equation led to his construction of the "horseshoe map," 17 and more generally contributed to the formulation of a broad research program in dynamics. ${ }^{18}$ Subsequently, Melnikov ${ }^{19}$ and Arnold ${ }^{20}$ provided rather general perturbative methods for proving the existence of homoclinic tangles such as those recognized by Poincaré and Smale.

This work, which was almost all done by mathematicians, brings us to the 1960s. In that decade, a few engineers and physical scientists became interested in chaos. Ueda's discovery in November 1961 was an early example, predating by 2 years Lorenz's better known paper on a strange (=chaotic) attractor in a truncated model for convection in a fluid layer. ${ }^{21}$ (Both Ueda and Lorenz acknowledged the importance of Birkhoff's work in enabling them to interpret their observations.) Throughout the 1960s Ueda continued to think about mathematical aspects of his findings, drawing on Levinson's work on second order ODEs as well as Poincaré's book. ${ }^{2}$ However, apart from a brief section in one paper [Ref. 22, §3.2, Figs. 6-8], some conference proceedings and a research report, ${ }^{23}$ he did not publish them for over 10 years. ${ }^{24}$ Lorenz's work also remained unnoticed by mathematicians until the 1970s, when J. A. Yorke was given a copy by a colleague in the Department of Meteorology at the University of Maryland, which he passed on to Smale. ${ }^{25}$ Soon thereafter, dynamical systems theory was percolating throughout the sciences and motivations and examples were flowing back to mathematics. By 1985, a bibliography of dynamical systems listed over 4400 papers and books. ${ }^{26}$

This brief history highlights only one thread within the rich tapestry of dynamical systems. More extensive treatments, along with comments on recent developments, can be found in Refs. 5 and 27, but advances have been so rapid and widespread that an adequate historical perspective on the past 50 years is still lacking. Chaos theory-as a part of nonlinear dynamics - has fostered a globally interconnected vision of the sciences in a time of strongly developing technologies. It has affected not only emerging interdisciplinary fields but also classical ones such as mechanics, within which the Kyoto Symposium was conceived and partially nurtured. In spite of some inflated claims and misuses of concepts and tools, which can augment but not replace careful mathematical modeling, the past fifty years of chaos have brought us much good sense, and a measure of order.

A detailed account of Poincaré's work on dynamics appears in Ref. 4. For more on the discoveries of Ueda and Lorenz, and their sometimes difficult paths to publication and acceptance, see Refs. 1 and 25. For a discussion of the sociological and cultural contexts of nonlinear dynamics and chaos, see Ref. 28.

\section{THE PAPERS IN THIS ISSUE}

The 13 papers that follow form a varied baker's dozen, representing several of the classical and more recent areas of nonlinear dynamics and ranging from basic theory to applied technology.

The mechanics of elastic structures and rigid bodies is treated in the papers of Lenci et al., ${ }^{29}$ Strzalko et al., ${ }^{30}$ and Kapitaniak et al. $^{31}$ Issues of mechanical modeling (e.g., of friction and impacts in Ref. 31), synchronization, control of chaos, imperfections, and symmetries of the mechanical systems play important roles in these studies. Lenci et al. exploit chaos properties to control the global nonlinear dynamics of simplified models of a large class of structures exhibiting interacting buckling phenomena, thus increasing their practical load carrying capacities. Strzalko et al. study synchronous behavior in an experimental set of two pairs of double pendula, with a view to converting base oscillations into rotational motions exploitable for energy production. Kapitaniak et al. present simulations and experiments on die throwing, addressing the theoretical predictability of 
outcomes in contrast with the difficulty of practical implementation.

Kreilos and Eckhardt ${ }^{32}$ investigate stability and bifurcations in transitional and weakly turbulent Couette flow. The model-the incompressible Navier-Stokes equation-is not in question here; the issue is to extract useful information from direct numerical simulations of a very highdimensional system, and by following branches of equilibria representing steady flow patterns, most of which are unstable. Chaotic saddles (homoclinic tangles) are found to produce transient turbulent bursts. Cvitanovič et al., ${ }^{33}$ building on earlier work on similar channel flows, show that a proper understanding of symmetries imposed by the governing equations is essential to visualizing and decomposing the global structure of high-dimensional state spaces.

Mathematical methods are developed in the paper of Sabuco et $a l .{ }^{34}$ and at substantially greater lengths in those of Bush et al., ${ }^{35}$ Lingala et al., ${ }^{36}$ and Budišić et al. ${ }^{37}$ Sabuco et al. extend their earlier work on safe sets in the context of chaos control to asymptotically safe sets, providing an algorithm that approximates the set of initial conditions that eventually enters a safe set. Bush et al. review an extensive program that uses algebraic-topological and combinatorial methods to deduce rigorous global information on iterated nonlinear maps, proving connections from saddle-type invariant sets to attracting sets. Lingala et al. describe particle filtering methods that approximate distributions of state variables observed in chaotic systems. Budišić et al. review the Koopman operator (an infinite-dimensional linear map that advances observable functions along orbits of a dynamical system) and explain how its eigenfunctions preserve global information; they also introduce continuous quantifications or ergodicity and mixing behaviors. These "datadriven" papers all illustrate theories and methods by means of multiple examples.

Hirata et al. ${ }^{38}$ revisit a data set collected from the giant axon of the squid (the preparation used in Hodgkin and Huxley's Nobel Prize winning work in which the dynamics of action potentials were first modeled). The authors show that a relaxed, numerically adapted version of Devaney's criteria for chaos ${ }^{39}$ identifies the neuron's voltage time series as chaotic, and also briefly describe a simple mapping that produces deterministic chaos in a neural network model of memory recall.

Finally, three papers describe interesting electronic systems with technological implications. Kohda et al. ${ }^{40}$ show that expanding attractors (originally, purely abstract mathematical objects) can be used to build analog-to-digital converters with high bit-rate accuracies. Sunada et al. ${ }^{41}$ take a complementary view, using a chaotically oscillating laser to produce bit sequences that pass stringent statistical tests for random number generators. In et $a l^{42}$ construct an integrated circuit containing an array of bistable oscillators that can be made to entrain to distinct frequency ranges, enabling a "circuit on a chip" to rapidly lock on components of an arbitrary radio-frequency spectrum.

While most of these papers were prepared independently (one pair ${ }^{30,31}$ does share three authors and another ${ }^{38,40}$ shares one), they nicely illustrate common interests in nonlinear dy- namics. In particular, computational methods (rigorously based, as in Refs. 35 and 37, or more formal) play an important part. This is likely to last for some time: 123 years after Poincaré's prize paper it is still embarassingly difficult to extract global information on ODEs defined in 3 or more dimensions, or invertible maps of 2 or more dimensions. Symmetries and bifurcations, cross sections and Poincaré maps are prevalent, and increasingly high dimensional phase spaces are being considered. Several papers discuss multiple applications, or present an approach or methods that apply beyond their specific examples, those of Bush et al., ${ }^{35}$ Cvitanovič et al., ${ }^{33}$ Hirata et al., ${ }^{38}$ Lingala et al., ${ }^{36}$ and Budišić et al. ${ }^{37}$ provide examples.

\section{ACKNOWLEDGMENTS}

The editors thank the International Union of Theoretical and Applied Mechanics for support of the IUTAM symposium on 50 Years of Chaos: Applied and Theoretical, held at Kyoto University November 28-December 2, 2011, at which preliminary versions of the papers included herein were first presented, and the AIP Chaos office, especially Linda Boniello, for assistance in assembling this focus issue.

${ }^{1}$ Y. Ueda, The Road to Chaos-II, 2nd ed. (Aerial, Santa Cruz, CA, 2001). ${ }^{2}$ H. J. Poincaré, Les méthodes nouvelles de la mécanique celeste (Gauthiers-Villars, Paris, 1892, 1893, 1899), Vols. 1-3. [English translation edited by D. Goroff, published by the American Institute of Physics, New York, 1993.]

${ }^{3}$ H. J. Poincaré, "Sur le problème des trois corps et les équations de la dynamique," Acta Math. 13, 1-270 (1890).

${ }^{4} \mathrm{~J}$. Barrow-Green, Poincaré and the Three Body Problem (American Mathematical Society, Providence, RI, 1997).

${ }^{5}$ F. Diacu and P. Holmes, Celestial Encounters: The Origins of Chaos and Stability (Princeton University Press, Princeton, NJ, 1996).

${ }^{6}$ G. D. Birkhoff, "Proof of Poincaré's geometric theorem," Trans. Am. Math. Soc. 14, 14-22 (1913).

${ }^{7}$ G. D. Birkhoff, Dynamical Systems (American Mathematical Society, Providence, RI, 1927) [Reprinted with an introduction by J. Moser and a preface by M. Morse, 1966.]

${ }^{8}$ G. D. Birkhoff, "Sur quelques courbes fermées remarquables," Bull. Soc. Math. France 60, 1-26 (1932).

${ }^{9}$ G. D. Birkhoff, "Sur l'existence de régions d'instabilité en dynamique," Ann. Inst. Henri Poincaré 2, 369-386 (1932).

${ }^{10}$ B. van der Pol and B. van der Mark, "Frequency demultiplication," Nature 120, 363-364 (1927).

${ }^{11}$ M. L. Cartwright and J. E. Littlewood, "On nonlinear differential equations of the second order. I: The equation $\ddot{y}+k\left(1-y^{2}\right) \dot{y}+y$ $=b \lambda k \cos (\lambda t+a), k$ large," J. Lond. Math. Soc. 20, 180-189 (1945).

${ }^{12}$ N. Levinson, "A second-order differential equation with singular solutions," Ann. Math. 50, 127-153 (1949).

${ }^{13}$ A. A. Andronov and L. Pontryagin, "Systèmes grossieres," Dokl. Akad. Nauk SSSR 14, 247-251 (1937).

${ }^{14}$ A. A. Andronov, A. A. Vitt, and S. E. Khaikin, Theory of Oscillators (translated by F. Immerzi from the Russian, 1st ed., Moscow, 1937) (Pergamon, London, UK, 1966).

${ }^{15}$ A. A. Andronov, E. A. Leontovich, I. I. Gordon, and A. G. Maier, Theory of Bifurcations of Dynamic Systems on a Plane (Israel Program for Scientific Translations, Jerusalem, 1971).

${ }^{16} \mathrm{~S}$. Smale, The Mathematics of Time (Springer, New York, 1980).

${ }^{17} \mathrm{~S}$. Smale, "Diffeomorphisms with many periodic points," in Differential and Combinatorial Topology: A Symposium in Honor of Marston Morse, edited by S. S. Cairns (Princeton University Press, Princeton, NJ, 1965), pp. $63-70$.

${ }^{18}$ S. Smale, "Differentiable dynamical systems," Bull. Am. Math. Soc. 73, 747-817 (1967). 
${ }^{19} \mathrm{~V}$. K. Melnikov, "On the stability of the center for time-periodic perturbations," Trans. Mosc. Math. Soc. 12, 1-57 (1963).

${ }^{20} \mathrm{~V}$. I. Arnold, "Instability of dynamical systems with several degrees of freedom," Sov. Math. Dokl. 5, 342-355 (1964).

${ }^{21}$ E. N. Lorenz, "Deterministic non-periodic flow," J. Atmos. Sci. 20, 130 141 (1963).

${ }^{22}$ C. Hayashi, Y. Ueda, N. Akamatsu, and H. Itakura, "On the behavior of self-oscillatory systems with external force," Trans. IECE 53-A(3), 150158 (1970). [Reprinted in Ref. 1.]

${ }^{23}$ C. Hayashi, Y. Ueda, and N. Akamatsu, "On steady-state solutions of a nonlinear differential equation of the second order," Res. Rep. RIMS (Kyoto Univ.) 113, 1-27 (1971) (in Japanese).

${ }^{24}$ Y. Ueda, N. Akamatsu, and C. Hayashi, "Computer simulation of nonlinear ordinary differential equations and non-periodic oscillations," Trans. IECE 56-A(4), 218-225 (1973). [Reprinted in Ref. 1.]

${ }^{25} \mathrm{E}$. N. Lorenz, The Essence of Chaos (University of Washington Press, Seattle, WA, 1993).

${ }^{26} \mathrm{~K}$. Shiraiwa, Bibiliography for Dynamical Systems (Department of Mathematics, Nagoya University, Japan, 1985).

${ }^{27} \mathrm{P}$. Holmes, "Ninety plus thirty years of nonlinear dynamics: More is different and less is more," Int. J. Bifurcation Chaos 15, 2703-2716 (2005).

${ }^{28}$ D. Aubin and A. Dahan Dalmedico, "Writing the history of dynamical systems and chaos: Longue durée and revolution, disciplines and cultures," Hist. Math. 29, 273-339 (2002).

${ }^{29}$ S. Lenci, D. Orlando, G. Rega, and P. B. Gonçalves, "Controlling practical stability and safety of mechanical systems by exploiting chaos properties," Chaos 22, 047502 (2012).

${ }^{30}$ J. Strzalko, J. Grabski, J. Wojewoda, M. Wiercigroch, and T. Kapitaniak, "Synchronous rotation of the set of double pendula: Experimental observations," Chaos 22, 047503 (2012).
${ }^{31}$ M. Kapitaniak, J. Strzalko, J. Grabski, and T. Kapitaniak, "The threedimensional dynamics of the die throw," Chaos 22, 047504 (2012).

${ }^{32}$ T. Kreilos and B. Eckhardt, "Periodic orbits near onset of chaos in plane Couette flow," Chaos 22, 047505 (2012).

${ }^{33}$ P. Cvitanovič, D. Borrero-Echeverry, K. M. Carroll, B. Robbins, and E. Siminos, "Cartography of high-dimensional flows: A visual guide to sections and slices," Chaos 22, 047506 (2012).

${ }^{34}$ J. Sabuco, M. A. F. Sanjuán, and J. A. Yorke, "Dynamics of partial control," Chaos 22, 047507 (2012).

${ }^{35}$ J. Bush, M. Gameiroy, S. Harker, H. Kokubu, K. Mischaikow, I. Obayashik, and P. Pilarczyk, "Combinatorial-topological framework for the analysis of global dynamics," Chaos 22, 047508 (2012).

${ }^{36}$ N. Lingala, N. Sri Namachchivaya, N. Perkowski, and H. C. Yeong, "Particle filtering in high-dimensional chaotic systems," Chaos 22, 047509 (2012).

${ }^{37}$ M. Budišić, R. Mohr, and I. Mezić, "Applied Koopmanism," Chaos 22, 047510 (2012).

${ }^{38}$ Y. Hirata, M. Oku, and K. Aihara, "Chaos in neurons and its application: Perspective of chaos engineering," Chaos 22, 047511 (2012).

${ }^{39}$ R. L. Devaney, An Introduction to Chaotic Dynamical Systems, 2 nd ed. (Westview, 2003).

${ }^{40}$ T. Kohda, Y. Horio, and K. Aihara, "Expansion attractors observed in A/D converters," Chaos 22, 047512 (2012).

${ }^{41}$ S. Sunada, T. Harayama, P. Davis, K. Tsuzuki, K. Arai, K. Yoshimura, and A. Uchida, "Noise amplification by chaotic dynamics in a delayed feedback laser system and its application to nondeterministic random bit generation," Chaos 22, 047513 (2012).

${ }^{42}$ V. In, P. Longhini, A. Kho, J. Neff, D. Leung, N. Liu, B. Meadows, F. Gordon, A. Bulsara, and A. Palacios, "Nonlinear channelizer," Chaos 22, 047514 (2012). 